BMJ Open

Diabetes

Research

\& Care

\section{Association of Mediterranean diet and cardiorespiratory fitness with the development of pre-diabetes and diabetes: the Coronary Artery Risk Development in Young Adults (CARDIA) study}

To cite: Bantle AE, Chow LS, Steffen LM, et al. Association of Mediterranean diet and cardiorespiratory fitness with the development of prediabetes and diabetes: the Coronary Artery Risk Development in Young Adults (CARDIA) study. BMJ Open Diabetes Research and Care 2016:4:e000229.

doi:10.1136/bmjdrc-2016000229

- Additional material is available. To view please visit the journal (http://dx.doi.org/ 10.1136/bmjdrc-2016000229).

Received 21 March 2016 Revised 24 June 2016 Accepted 21 July 2016

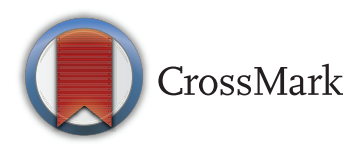

For numbered affiliations see end of article.

Correspondence to Dr Anne E Bantle; bant0015@umn.edu

\section{ABSTRACT}

Objective: To better understand the association between a modified Mediterranean diet pattern in young adulthood, cardiorespiratory fitness in young adulthood, and the odds of developing pre-diabetes or diabetes by middle age.

Research design and methods: Participants from the Coronary Artery Risk Development in Young Adults (CARDIA) study who did not have pre-diabetes or diabetes at baseline (year 0 (Y0), ages 18-30) and who had data available at the Y0 and year 25 (Y25) visits were included in this analysis $(n=3358)$. Polytomous logistic regression models were used to assess the association between baseline dietary intake and fitness data and odds of pre-diabetes or diabetes by middle age (Y25, ages 43-55).

Results: At the Y25 visit, 1319 participants (39\%) had pre-diabetes and 393 (12\%) had diabetes. Higher baseline fitness was associated with lower odds of prediabetes and of diabetes at Y25. After adjustment for covariates, each SD increment in treadmill duration (181 s) was associated with lower odds for prediabetes (OR $0.85,95 \% \mathrm{Cl} 0.75$ to $0.95, \mathrm{p}=0.005$ ) and for diabetes (OR $0.71,95 \% \mathrm{Cl} 0.60$ to $0.85, \mathrm{p}=0.0002$ ) when compared to normal glycemia. A modified Mediterranean diet pattern was not associated with either pre-diabetes or diabetes. No interaction between cardiorespiratory fitness and dietary intake was observed, but baseline fitness remained independently associated with incident pre-diabetes and diabetes following adjustment for diet.

Conclusions: Higher cardiorespiratory fitness in young adulthood, but not a modified Mediterranean diet pattern, is associated with lower odds of prediabetes and of diabetes in middle age.

Trial registration number: NCT00005130.

\section{INTRODUCTION}

The increasing worldwide prevalence of type 2 diabetes mellitus underscores the need for

\section{Key messages}

Higher cardiorespiratory fitness in young adulthood was associated with lower odds for prediabetes and for diabetes in middle age, regardless of diet.

- The association between a modified Mediterranean diet pattern score and prediabetes or diabetes was not statistically significant after controlling for fitness.

- Strategies targeting the prevention of diabetes should emphasize the beneficial contribution of cardiorespiratory fitness.

effective diabetes prevention strategies. The Mediterranean lifestyle, a combination of Mediterranean diet and physical activity, has attracted interest in this regard.

The Mediterranean diet is characterized by a high intake of vegetables, legumes, fruit, nuts, unrefined grains, fish, and monounsaturated fatty acids (MUFAs), primarily in the form of olive oil. This is combined with a low intake of meat and poultry and moderate consumption of alcohol, especially red wine. This dietary pattern has been associated with lower odds of mortality ${ }^{1}$ and reduced incidence of type 2 diabetes in observational studies. ${ }^{2}{ }^{3}$ In a randomized, controlled clinical trial, adoption of a Mediterranean diet with additional supplementation of olive oil reduced diabetes incidence in people at high risk of cardiovascular disease. ${ }^{4}$ There is also evidence of improved glucose metabolism in healthy people ${ }^{6}$ and people with type 2 diabetes $^{7}{ }^{8}$ who consume a Mediterranean diet.

Higher cardiorespiratory fitness has likewise been associated with reduced incidence 
of type 2 diabetes. ${ }^{9-14}$ While cardiorespiratory fitness can be increased by physical activity, ${ }^{15}$ a large component of cardiorespiratory fitness is also determined by other factors, such as genetics. ${ }^{16}$ Physical activity is often used in interventions to reduce the incidence of type 2 diabetes, including the intervention studied in the Diabetes Prevention Program. ${ }^{17} 18$ A Mediterranean diet combined with physical activity has been associated with lower mortality in a general population ${ }^{19}$ and improved outcomes in patients with type 2 diabetes. ${ }^{20}$ However, it remains unknown how the Mediterranean diet and cardiorespiratory fitness may complement each other, and specifically whether Mediterranean diet and cardiorespiratory fitness interact to jointly influence the odds of future pre-diabetes or diabetes.

The purpose of this analysis was to examine the relationship between a modified Mediterranean diet pattern score in young adulthood, cardiorespiratory fitness in young adulthood, and the odds of developing prediabetes or type 2 diabetes later in life. The Coronary Artery Risk Development in Young Adults (CARDIA) study has collected data over 25 years in a cohort of participants who were young adults in 1985-1986, including data on dietary intake and cardiorespiratory fitness. Thus, it provided a robust data set for this analysis. We hypothesized that fitness and dietary intake are not independent and that consumption of a Mediterranean diet and high fitness in young adulthood would jointly reduce the odds of pre-diabetes and of diabetes in middle age.

\section{RESEARCH DESIGN AND METHODS}

The CARDIA study is a prospective, multicenter, longitudinal cohort study designed to investigate coronary heart disease risk factors in young adults. Participants were recruited from four US study centers (Minneapolis, Minnesota; Chicago, Illinois; Birmingham, Alabama; and Oakland, California) with recruitment balanced by age, race, sex, and education level. A total of 5115 black and white women and men aged 18-30 years were enrolled in 1985-1986. Study visits were at year 0 (Y0) and again at years $2,5,7,10,15,20$, and 25 after enrollment. Retention rates were $91 \%, 86 \%, 81 \%, 79 \%, 74 \%$, $72 \%$, and $72 \%$ of the surviving cohort at each visit, respectively. Additional details of the CARDIA study methods have been published elsewhere. ${ }^{21}$ All study procedures were approved by the institutional review board from each study center, and all participants provided written informed consent for study participation.

For the purposes of this analysis, only data from Y0 and year 25 (Y25) were included. Participants were excluded if they had diabetes or pre-diabetes at Y0 $(n=138)$, if they did not have fitness or dietary intake data available at $\mathrm{Y0}(\mathrm{n}=67 ; \mathrm{n}=64 \mathrm{did}$ not have fitness data and $n=4$ did not have dietary intake data), or if their diabetes status at Y25 was unknown $(\mathrm{n}=1552)$. This yielded 3358 participants for this analysis.

\section{Fitness assessment}

Fitness was assessed by symptom-limited, graded treadmill exercise duration at Y0. This was measured using a modified Balke protocol, consisting of nine stages of progressively increasing difficulty (2 min each, maximum of $18 \mathrm{~min}$ total) ${ }^{22}$ Fitness data are presented as total duration of treadmill exercise before a participant stopped exercise due to symptoms, in seconds. Longer duration indicates higher cardiorespiratory fitness.

\section{Physical activity assessment}

Physical activity data over the prior year were collected at the Y0 visit. Data were self-reported by study participants and were collected by an interviewer-administered questionnaire. ${ }^{23}$ Data were measured in exercise units, with 300 exercise units approximately equal to $150 \mathrm{~min}$ of moderate physical activity per week. ${ }^{24}$

\section{Diet assessment}

The CARDIA dietary history questionnaire was administered at Y0 by trained interviewers. ${ }^{25}$ The questionnaire was designed specifically for the CARDIA study, and dietary intake obtained was for the month prior to the interview. $^{25}$ The reliability and validity of the diet history method were evaluated and found to be acceptable, although the results were found to be less reliable in black participants. ${ }^{26}$

We developed a diet score that characterized a modified Mediterranean dietary pattern. Our diet score was developed a priori and was adapted from a previously published Mediterranean diet score $^{127}$ for use in an American young adult population. A similarly adapted score has been used in the CARDIA cohort to characterize a Mediterranean-like dietary pattern. ${ }^{28}$ We refer to our diet score as the Americanized Mediterranean diet score (AmMedDiet score).

To calculate the AmMedDiet score, sex-specific median intake was determined for 15 food groups created from the individual foods reported by CARDIA participants. We assigned one point for intake at or above the sex-specific median, and zero points for intake below the median, for food groups representative of an Americanized Mediterranean diet (vegetables, legumes, fruit and nuts, fish and seafood, whole grains, eggs, milk, and beneficial fat ratio). Beneficial fat intake was estimated by the sum of MUFA plus polyunsaturated fatty acids (PUFA), divided by saturated fatty acids (SFA) ((MUFA+PUFA)/SFA ratio), as previously described. ${ }^{28}$ This differs from the estimate of beneficial fat intake in the originally published Mediterranean diet score, which used the MUFA/SFA ratio. ${ }^{1}$ This change was necessary because unlike in a Greek population, olive oil was not typically consumed in the American diet during 1985-1986 and most beneficial fat intake was from other sources. Also, although high dairy consumption is traditionally not considered a part of the Mediterranean diet, we positively scored high dairy intake in our 
AmMedDiet score because of previous data associating dairy consumption with lower odds of insulin resistance and type 2 diabetes, including in the CARDIA cohort. ${ }^{29}$ In the traditional Mediterranean diet, dairy intake is mostly from high fat products such as cheese and yogurt, ${ }^{1}$ which differs from the dairy consumption pattern of the CARDIA cohort in the USA in 1985$1986 .{ }^{29}$ The final difference between our score and the previously published Mediterranean diet score $^{1}$ is the addition of separate categories for snacks, sweets, beverages, potatoes, eggs, and refined grains in our score, added to be inclusive of all food groups represented in the CARDIA dietary intake data set.

In our score, zero points were assigned for intake at or above the sex-specific median, and one point was assigned for intake below the median, for food groups not consistent with an Americanized Mediterranean diet (meat and poultry, refined grains, potatoes, snacks, sweets, and beverages). Alcohol intake was also included in the score, with moderate alcohol intake scored positively. One point was given for daily alcohol intake of 10-50 $\mathrm{g}$ for men and 5-25 $\mathrm{g}$ for women, and zero points were given for consumption below or above these ranges. Thus, our diet score ranged from 0 (least similar to an Americanized Mediterranean dietary intake pattern) to 15 (most similar to an Americanized Mediterranean dietary intake pattern).

\section{Definition of pre-diabetes and diabetes}

Blood was drawn at study visits according to a standard protocol. ${ }^{21}$ Serum glucose concentrations were measured using the hexokinase method at Linco Research (St Charles, Missouri, USA). Hemoglobin A1c (HgbA1c) was determined at the University of Minnesota (Minneapolis, Minnesota, USA) using the Tosoh G7 high-performance liquid chromatography instrument. Medications were self-reported at each study visit.

Pre-diabetes was defined by one of the following features at the Y25 visit: fasting glucose of $100-125 \mathrm{mg} / \mathrm{dL}$, 2-hour glucose during a $75 \mathrm{~g}$ oral glucose tolerance test of $140-199 \mathrm{mg} / \mathrm{dL}$, or HgbAlc of $5.7-6.4 \%$ $(39-46 \mathrm{mmol} / \mathrm{mol})$. Diabetes was defined by one of the following features at the Y25 visit: fasting glucose $\geq 126 \mathrm{mg} / \mathrm{dL}$, self-reported use of medications for the treatment of diabetes mellitus, 2-hour glucose during the oral glucose tolerance test of $\geq 200 \mathrm{mg} / \mathrm{dL}$, or HgbAlc $\geq 6.5 \%(48 \mathrm{mmol} / \mathrm{mol})$. Incidence of prediabetes or diabetes at Y25 was the primary outcome.

\section{Models and statistical analysis}

Polytomous logistic regression analyses were used to examine the association of Y0 fitness measured by treadmill duration, Y0 AmMedDiet score, and incidence of pre-diabetes or incidence of diabetes at Y25. This modeling approach was selected to allow pre-diabetes and diabetes to be considered in the same model. We first tested the models with ordinal logistic regression to assess the proportional odds assumption whether the associations of Y0 treadmill duration and Y0 AmMedDiet score were statistically significantly the same for prediabetes compared to normal glycemia and diabetes compared to normal glycemia. Since the proportional odds assumption was rejected, we ran the same models using polytomous logistic regression, presenting separate ORs for incident pre-diabetes and incident diabetes at Y25.

We developed three polytomous logistic regression models. The first included the Y0 AmMedDiet score and was designed to test the relationship between Y0 diet and odds of pre-diabetes or diabetes at Y25. The second model included Y0 cardiorespiratory fitness measured by treadmill duration and was designed to test the relationship between Y0 fitness and odds of pre-diabetes or diabetes at Y25. The third model included the Y0 AmMedDiet score and Y0 treadmill duration, with the purpose of analyzing diet and fitness simultaneously in the same model to look for a joint reduction in odds of pre-diabetes or diabetes at Y25.

All models were adjusted for age, sex, race, field center, body mass index (BMI), education (highest year of school completed), smoking (current/former/ never), caloric intake (kcal/day), and self-reported physical activity. All covariate data used for adjustment were from Y0. The interaction between AmMedDiet score and treadmill duration was not statistically significant once the third model was adjusted for covariates, indicating that there was not a joint reduction in odds of prediabetes or diabetes when diet and fitness were analyzed in the same model ( $p$-value of type III test for the interaction between the AmMedDiet score and treadmill duration was 0.11 ). For this reason, the interaction term was not included in the adjusted version of this model. The interaction between the AmMedDiet score and treadmill duration was also statistically nonsignificant when modeled in tertiles. Statistical analyses were performed using Statistical Analysis Software (V.9.3, SAS Institute, Cary, North Carolina, USA).

\section{RESULTS}

Three thousand three hundred and fifty-eight participants from the CARDIA cohort were included in these analyses. Participant characteristics at baseline (Y0) are summarized in table 1 . Mean age at baseline was 25 years, mean weight 156.2 pounds, and mean BMI $24.4 \mathrm{~kg} / \mathrm{m}^{2}$. At the Y25 visit, 1319 participants had prediabetes and 393 had diabetes. Participants who developed diabetes at Y25 were heavier at Y0, more likely to be black than white, and more likely to be female compared to those with normal glucose and HgbAlc. Participants with pre-diabetes at Y25 were also heavier, had a higher proportion of blacks, and had equal numbers of men and women at baseline compared to those with normal glucose and HgbAlc. Participant characteristics stratified by tertiles of cardiorespiratory fitness are presented in online supplementary table S1. 
Table 1 Participant characteristics at baseline (Y0) stratified by $Y 25$ outcome

\begin{tabular}{|c|c|c|c|c|}
\hline & $\begin{array}{l}\text { Absence of pre-diabetes or } \\
\text { diabetes at Y25 }(n=1646)\end{array}$ & $\begin{array}{l}\text { Pre-diabetes at } \\
\text { Y25 }(n=1319)\end{array}$ & $\begin{array}{l}\text { Diabetes at } \\
\text { Y25 }(n=393)\end{array}$ & $\begin{array}{l}\text { p Value from } \\
\text { ANOVA F-test } \\
\text { or } \chi^{2} \text { test }\end{array}$ \\
\hline Age (years) & $24.8(3.6)$ & $25.3(3.6)$ & $25.5(3.6)$ & $<0.0001$ \\
\hline BMI $\left(\mathrm{kg} / \mathrm{m}^{2}\right)$ & $23.4(4.3)$ & $24.5(4.6)$ & $28.2(5.8)$ & $<0.0001$ \\
\hline Weight (Ibs) & $148.9(31.6)$ & $158.1(32.9)$ & $180.0(41.8)$ & $<0.0001$ \\
\hline \multicolumn{5}{|l|}{ Race } \\
\hline Black & $36 \%$ & $54 \%$ & $69 \%$ & $<0.0001$ \\
\hline White & $64 \%$ & $46 \%$ & $31 \%$ & \\
\hline \multicolumn{5}{|l|}{ Sex } \\
\hline Male & $37 \%$ & $50 \%$ & $45 \%$ & $<0.0001$ \\
\hline Female & $63 \%$ & $50 \%$ & $55 \%$ & \\
\hline Treadmill duration (s) & $604(176)$ & $589(183)$ & $508(176)$ & $<0.0001$ \\
\hline $\begin{array}{l}\text { Self-reported physical } \\
\text { activity (exercise units) }\end{array}$ & $424(286)$ & $424(308)$ & $385(294)$ & 0.053 \\
\hline $\begin{array}{l}\text { AmMedDiet score } \\
\text { (range 0-15) }\end{array}$ & $7.1(2.1)$ & $6.9(2.1)$ & $6.5(1.9)$ & $<0.0001$ \\
\hline
\end{tabular}

There was an association between a higher AmMedDiet score at Y0 and lower odds of Y25 prediabetes and Y25 diabetes before adjustment for covariates in the first model (table 2). This association did not maintain statistical significance for either pre-diabetes or diabetes when the model was fully adjusted.

Longer treadmill duration at $\mathrm{Y0}$ was linearly associated with lower odds of Y25 pre-diabetes and of Y25 diabetes in the second model (table 2). This association was maintained in the fully adjusted model, such that for each SD (181 s) higher treadmill duration at Y0 there were $15 \%$ lower odds of Y25 pre-diabetes (OR $0.85,95 \%$ CI 0.75 to $0.95, \mathrm{p}=0.005)$ and $29 \%$ lower odds of diabetes (OR $0.71,95 \%$ CI 0.60 to 0.85 , $\mathrm{p}=0.0002$ ).

We found a correlation between cardiorespiratory fitness and physical activity $(r=0.39, \mathrm{p}<0.0001)$. Given this correlation, we also ran our polytomous logistic regression model using physical activity in place of cardiorespiratory fitness. This did not show a statistically significant association between Y0 physical activity and Y25 outcome after adjustment for covariates (see online supplementary table $\mathrm{S} 2$ ).

When dietary intake and fitness were analyzed simultaneously in the third model, the association between fitness and Y25 outcome did not change (table 3). In the fully adjusted model, each SD (181 s) increment for Y0 treadmill duration was associated with $15 \%$ lower odds of Y25 pre-diabetes (OR $0.85,95 \%$ CI 0.75 to 0.95 , $\mathrm{p}=0.005$ ) and $28 \%$ lower odds of diabetes (OR 0.72, $95 \%$ CI 0.60 to $0.86, p=0.0003)$. The association with dietary intake, on the other hand, was no longer statistically significant after accounting for fitness.

The third model, including dietary intake and fitness simultaneously, was further analyzed by tertiles of Y0 AmMedDiet score and Y0 treadmill duration (see online supplementary table S3). This demonstrates that the lowest ORs for Y25 pre-diabetes or diabetes were seen in the highest tertile of $\mathrm{Y} 0$ fitness when compared to reference.

Table 2 Association of YO AmMedDiet score or YO treadmill duration (analyzed separately) and ORs of pre-diabetes or diabetes at Y25

\begin{tabular}{|c|c|c|c|c|}
\hline & Y25 pre-diabetes & p Value & Y25 diabetes & p Value \\
\hline \multicolumn{5}{|c|}{ YO AmMedDiet score (per 1 SD (2.1 point) increment) } \\
\hline Unadjusted & $0.91(0.85$ to 0.98$)$ & 0.01 & $0.78(0.69$ to 0.87$)$ & $<0.0001$ \\
\hline Adjusted $^{*}$ & $1.00(0.92$ to 1.08$)$ & 0.96 & $0.90(0.79$ to 1.03$)$ & 0.13 \\
\hline \multicolumn{5}{|c|}{ Y0 treadmill duration (per $1 \mathrm{SD}$ (181.0 s) increment) } \\
\hline Unadjusted & $0.92(0.86$ to 0.99$)$ & 0.03 & $0.59(0.53$ to 0.66$)$ & $<0.0001$ \\
\hline Adjusted* & $0.85(0.75$ to 0.95$)$ & 0.005 & $0.71(0.60$ to 0.85$)$ & 0.0002 \\
\hline
\end{tabular}

${ }^{*}$ Adjusted for age, sex, race, field center, physical activity, BMI, education, smoking, and caloric intake.

Data are ORs $(95 \%$ Cls $)$.

AmMedDiet, Americanized Mediterranean diet; BMI, body mass index; Y0, year 0; Y25, year 25. 
Table 3 Association of YO AmMedDiet score and treadmill duration (analyzed simultaneously) and ORs of pre-diabetes or diabetes at Y25

\begin{tabular}{|c|c|c|c|c|}
\hline & Y25 Pre-diabetes & p Value & Y25 diabetes & p Value \\
\hline $\begin{array}{l}\text { YO AmMedDiet score and Y0 treadmill duration, unadjuste } \\
\text { YO AmMedDiet score (per } 1 \text { SD (2.1 point) increment) } \\
\text { YO treadmill duration (per } 1 \text { SD (181.0 s) increment) }\end{array}$ & $\begin{array}{l}0.92(0.86 \text { to } 0.99) \\
0.93(0.86 \text { to } 1.00)\end{array}$ & $\begin{array}{l}0.03 \\
0.06\end{array}$ & $\begin{array}{l}0.83(0.74 \text { to } 0.93) \\
0.61(0.54 \text { to } 0.68)\end{array}$ & $\begin{array}{c}0.001 \\
<0.0001\end{array}$ \\
\hline $\begin{array}{l}\text { YO AmMedDiet score and Y0 treadmill duration, adjusted* } \\
\text { YO AmMedDiet score (per } 1 \text { SD (2.1 point) increment) } \\
\text { Y0 treadmill duration (per } 1 \text { SD (181.0 s) increment) }\end{array}$ & $\begin{array}{l}1.01 \text { (0.93 to } 1.09) \\
0.85(0.75 \text { to } 0.95)\end{array}$ & $\begin{array}{l}0.84 \\
0.005\end{array}$ & $\begin{array}{l}0.92(0.81 \text { to } 1.05) \\
0.72(0.60 \text { to } 0.86)\end{array}$ & $\begin{array}{l}0.21 \\
0.0003\end{array}$ \\
\hline
\end{tabular}

\section{CONCLUSIONS}

This study examined the relationship between a modified Mediterranean diet score and cardiorespiratory fitness in young adulthood and the odds of developing pre-diabetes or diabetes by middle age. Using polytomous logistic regression models and data in CARDIA study participants, we found that high baseline cardiorespiratory fitness was associated with lower odds of prediabetes and lower odds of diabetes 25 years later; this association did not differ by baseline diet. We also found no significant association between a modified Mediterranean diet score and the development of prediabetes or diabetes 25 years later after adjustment for baseline covariates, including fitness level.

The findings presented here are consistent with previous data linking greater cardiorespiratory fitness to lower odds of future diabetes. ${ }^{9-14}{ }^{30}$ Furthermore, our findings suggest that fitness level may be a confounding factor in studies associating dietary intake with reduced odds of diabetes, and highlight the importance of adjusting for fitness in such analyses. While many studies have examined the relationship between dietary intake or fitness and odds of future diabetes, few have looked at fitness and dietary intake together in the same cohort. This makes our analysis distinct.

The mechanisms through which fitness leads to reduced type 2 diabetes incidence remain incompletely understood. Contributing mechanisms may include improvement in muscle and liver insulin sensitivity, changes in fat oxidation and in visceral fat accumulation and body composition, reduction in systemic inflammation, and generalized weight loss. ${ }^{14} 3031$ In this study, we observed a statistically significant association between fitness and odds of diabetes that persisted even after correction for baseline BMI, which highlights the value of fitness even if an individual is overweight or obese. Our data also suggest that the highest level of baseline fitness is associated with the most benefit. Thus, the mechanism by which fitness is related to future pre-diabetes or diabetes risk may depend at least in part on fitness level.

Given that physical activity has an established doseresponse relationship to cardiorespiratory fitness, we also assessed the contribution of physical activity by including a model comparing Y0 self-reported physical activity with Y25 outcome in our analysis (see online supplementary table S2). This model did not show a statistically significant association between $\mathrm{Y} 0$ physical activity level and Y25 diabetes or pre-diabetes after adjustment for covariates. However, we acknowledge that this lack of significance may reflect the limitations of self-reported data and be due to reporting rather than a true absence of association, a limitation that may also apply to the dietary intake data available from the CARDIA cohort.

A main strength of our analysis is the use of the robust data set provided by the CARDIA study, which includes measures of fitness, physical activity, dietary intake, and diabetes status and follow-up over 25 years. Another study strength is the use of polytomous logistic regression modeling, which allowed concurrent examination of prediabetes and diabetes in middle age. A limitation is that this analytic technique does not lend itself to time-to-event analyses. To address this, we repeated our models with Y20 data in place of Y0 data, which did not change any of the associations reported here, but did significantly reduce sample size due to cohort attrition over time. There was a strong correlation between Y0 and Y20 treadmill duration (Pearson correlation coefficient 0.69, $\mathrm{p}<0.0001)$ as well as Y0 and Y20 AmMedDiet score (Pearson correlation coefficient $0.30, \mathrm{p}<0.0001$ ). Another limitation is that factors, such as smoking and parity, may have changed over time and influenced the association of baseline dietary intake and fitness with outcome. Nonetheless, our study design allowed for comparison of dietary intake with 25-year outcome, one of the longest studies of this type to the best of our knowledge.

There are limitations of our AmMedDiet pattern score. First, foods available in the marketplace have changed over 25 years, and the typical American diet has changed over time. In the 1980s, Americans were less likely to be aware of or to consume a Mediterranean diet. Our AmMedDiet score was designed to take this into account, but it is possible that the score did not appropriately capture a true Mediterranean-like diet in this cohort of American young adults. In particular, the 
score used the (MUFA+PUFA)/SFA ratio to estimate beneficial fat intake in place of a direct estimate of olive oil intake. In the CARDIA cohort, a large proportion of MUFA intake came from meat rather than olive oil, which may contribute to the weak association between young adult AmMedDiet score and middle-aged diabetes outcome. It has been postulated that MUFAs from olive oil are a key component of the Mediterranean diet leading to protection from diabetes ${ }^{5} 3233$ and that oleate is an MUFA that may be of particular benefit in mitigating cardiovascular risk and insulin resistance. ${ }^{34}$ In addition, non-MUFA components of olive oil, such as phenolic compounds, may provide benefit. ${ }^{35}$ Another limitation is the use of sex-specific population medians for food group cut-offs in the score, and the modification of the score from the original Mediterranean diet score. ${ }^{1}$ This design was chosen to tailor our score to the CARDIA cohort and is based on a previously published score from the CARDIA literature. ${ }^{28} \mathrm{~A}$ disadvantage is that this design limits comparison of our findings using this score to other population cohorts. Finally, the choice of a composite score means that we cannot comment on individual foods groups which may be important for risk of incident diabetes.

The findings of this study are clinically relevant. The current epidemic of obesity and type 2 diabetes underscores the need for new diabetes prevention strategies, focusing on prevention of pre-diabetes and halting progression from pre-diabetes to diabetes. Our data did not show a relationship between baseline Mediterranean diet pattern or physical activity and odds of pre-diabetes and diabetes 25 years later. We do show that baseline cardiorespiratory fitness is an independent predictor of odds of pre-diabetes and diabetes. Caution must be exercised in extrapolating this result to a recommendation to increase cardiorespiratory fitness through physical activity, as genetics has been shown to play an important role in baseline cardiorespiratory fitness and in individual response to physical activity. ${ }^{16}$ Nonetheless, a doseresponse relationship has been shown between increased physical activity through structured exercise programs and increased cardiorespiratory fitness. ${ }^{15}$

In conclusion, in this prospective, longitudinal cohort study of participants followed over 25 years, higher cardiorespiratory fitness in young adulthood was associated with lower odds of pre-diabetes and lower odds of diabetes in middle age. There was no association with a modified Mediterranean diet pattern or with physical activity when accounting for covariates and for fitness. Consequently, strategies targeting the prevention of prediabetes and type 2 diabetes should consider the contribution of cardiorespiratory fitness.

\section{Author affiliations}

${ }^{1}$ Division of Endocrinology and Diabetes, Department of Medicine, University of Minnesota, Minneapolis, Minnesota, USA

${ }^{2}$ Division of Epidemiology and Community Health, School of Public Health, University of Minnesota, Minneapolis, Minnesota, USA
${ }^{3}$ Clinical and Translational Science Institute, University of Minnesota, Minneapolis, Minnesota, USA

${ }^{4}$ Division of Biostatistics, School of Public Health, University of Minnesota, Minneapolis, Minnesota, USA

${ }^{5}$ Division of Pediatrics and Adolescent Medicine, Department of Pediatrics, University of Alabama at Birmingham, Birmingham, Alabama, USA

${ }^{6}$ Department of Exercise Science and Sport Management, Kennesaw State University, Kennesaw, Georgia, USA

${ }^{7}$ Division of Cardiovascular Sciences, National Heart, Lung, and Blood Institute, Bethesda, Maryland, USA

Acknowledgements This manuscript has been reviewed by CARDIA for scientific content.

Contributors LSC, PJS, LMS, and AEB designed the research. QW, JH, LSC, PJS, and AEB analyzed the data. AEB, LSC, and PJS wrote the manuscript. All authors contributed to discussion and to review of the manuscript. $A E B$ has primary responsibility for the final content.

Funding The Coronary Artery Risk Development in Young Adults Study (CARDIA) is conducted and supported by the National Heart, Lung, and Blood Institute (NHLBI) in collaboration with the University of Alabama at Birmingham (HHSN268201300025C and HHSN268201300026C),

Northwestern University (HHSN268201300027C), University of Minnesota (HHSN268201300028C), Kaiser Foundation Research Institute (HHSN268201300029C), and Johns Hopkins University School of Medicine (HHSN268200900041C). CARDIA is also partially supported by the Intramural Research Program of the National Institute on Aging (NIA) and an intra-agency agreement between NIA and NHLBI (AG0005). This work was sponsored in part by the National Institutes of Health (NIH) grant T32 DK007203 (AEB).

\section{Competing interests None declared.}

Ethics approval Institutional Review Board at each participating institution.

Provenance and peer review Not commissioned; externally peer reviewed.

Data sharing statement No additional data are available.

Open Access This is an Open Access article distributed in accordance with the Creative Commons Attribution Non Commercial (CC BY-NC 4.0) license, which permits others to distribute, remix, adapt, build upon this work noncommercially, and license their derivative works on different terms, provided the original work is properly cited and the use is non-commercial. See: http:// creativecommons.org/licenses/by-nc/4.0/

\section{REFERENCES}

1. Trichopoulou A, Costacou T, Bamia C, et al. Adherence to a Mediterranean diet and survival in a Greek population. $N$ Engl J Med 2003;348:2599-608.

2. Martinez-Gonzalez MA, de la Fuente-Arrillaga $C$, Nunez-Cordoba $\mathrm{JM}$, et al. Adherence to Mediterranean diet and risk of developing diabetes: prospective cohort study. BMJ 2008;336:1348-51.

3. Esposito $\mathrm{K}$, Chiodini $\mathrm{P}$, Maiorino $\mathrm{MI}$, et al. Which diet for prevention of type 2 diabetes? A meta-analysis of prospective studies. Endocrine 2014;47:107-16.

4. Salas-Salvado J, Bullo M, Babio N, et al. Reduction in the incidence of type 2 diabetes with the Mediterranean diet: results of the PREDIMED-Reus nutrition intervention randomized trial. Diabetes Care 2011;34:14-19.

5. Salas-Salvado J, Bullo M, Estruch R, et al. Prevention of diabetes with Mediterranean diets: a subgroup analysis of a randomized trial. Ann Intern Med 2014;160:1-10.

6. Perez-Jimenez F, Lopez-Miranda J, Pinillos MD, et al. A Mediterranean and a high-carbohydrate diet improve glucose metabolism in healthy young persons. Diabetologia 2001;44:2038-43.

7. Esposito K, Maiorino MI, Ciotola M, et al. Effects of a Mediterranean-style diet on the need for antihyperglycemic drug therapy in patients with newly diagnosed type 2 diabetes: a randomized trial. Ann Intern Med 2009;151:306-14.

8. Esposito K, Maiorino MI, Petrizzo M, et al. The effects of a Mediterranean diet on the need for diabetes drugs and remission of 
newly diagnosed type 2 diabetes: follow-up of a randomized trial. Diabetes Care 2014;37:1824-30.

9. Lynch J, Helmrich SP, Lakka TA, et al. Moderately intense physical activities and high levels of cardiorespiratory fitness reduce the risk of non-insulin-dependent diabetes mellitus in middle-aged men. Arch Intern Med 1996;156:1307-14.

10. Hu FB, Sigal RJ, Rich-Edwards JW, et al. Walking compared with vigorous physical activity and risk of type 2 diabetes in women: a prospective study. JAMA 1999;282:1433-9.

11. Wei M, Gibbons LW, Mitchell TL, et al. The association between cardiorespiratory fitness and impaired fasting glucose and type 2 diabetes mellitus in men. Ann Intern Med 1999;130:89-96.

12. Sawada SS, Lee IM, Muto T, et al. Cardiorespiratory fitness and the incidence of type 2 diabetes: prospective study of Japanese men. Diabetes Care 2003;26:2918-22.

13. Sui X, Hooker SP, Lee IM, et al. A prospective study of cardiorespiratory fitness and risk of type 2 diabetes in women. Diabetes Care 2008;31:550-5.

14. Carnethon MR, Sternfeld B, Schreiner PJ, et al. Association of 20-year changes in cardiorespiratory fitness with incident type 2 diabetes: the coronary artery risk development in young adults (CARDIA) fitness study. Diabetes Care 2009;32:1284-8.

15. Church TS, Earnest CP, Skinner JS, et al. Effects of different doses of physical activity on cardiorespiratory fitness among sedentary, overweight or obese postmenopausal women with elevated blood pressure: a randomized controlled trial. JAMA 2007;297:2081-91.

16. Bouchard C, Sarzynski MA, Rice TK, et al. Genomic predictors of the maximal $\mathrm{O}(2)$ uptake response to standardized exercise training programs. J Appl Physiol 2011;110:1160-70.

17. Knowler WC, Barrett-Connor E, Fowler SE, et al. Reduction in the incidence of type 2 diabetes with lifestyle intervention or metformin. N Engl J Med 2002;346:393-403.

18. Bray GA, Chatellier A, Duncan C, et al. 10-year follow-up of diabetes incidence and weight loss in the Diabetes Prevention Program Outcomes Study. Lancet 2009;374:1677-86.

19. Knoops KB, de Groot LM, Kromhout D, et al. Mediterranean diet, lifestyle factors, and 10-year mortality in elderly European men and women: the hale project. JAMA 2004;292:1433-9.

20. Toobert DJ, Glasgow RE, Strycker LA, et al. Biologic and quality-of-life outcomes from the Mediterranean lifestyle program: a randomized clinical trial. Diabetes Care 2003;26:2288-93.
21. Friedman GD, Cutter GR, Donahue RP, et al. CARDIA: study design, recruitment, and some characteristics of the examined subjects. J Clin Epidemiol 1988;41:1105-16.

22. Sidney S, Haskell WL, Crow R, et al. Symptom-limited graded treadmill exercise testing in young adults in the CARDIA study. Med Sci Sports Exerc 1992;24:177-83.

23. Jacobs DR, Hahn LP, Haskell WL, et al. Validity and reliability of short physical activity history: CARDIA and the Minnesota Heart Health Program. J Cardiopulm Rehabil 1989;9:448-59.

24. Gabriel KP, Sidney S, Jacobs DR, et al. Convergent validity of a brief self-reported physical activity questionnaire. Med Sci Sports Exerc 2014;46:1570-7.

25. McDonald A, Van Horn L, Slattery M, et al. The CARDIA dietary history: development, implementation, and evaluation. J Am Diet Assoc 1991;91:1104-12.

26. Liu K, Slattery M, Jacobs D Jr, et al. A study of the reliability and comparative validity of the cardia dietary history. Ethn Dis 1994;4:15-27.

27. Trichopoulou A, Kouris-Blazos A, Wahlqvist ML, et al. Diet and overall survival in elderly people. BMJ 1995;311:1457-60.

28. Steffen LM, Van Horn L, Daviglus ML, et al. A modified Mediterranean diet score is associated with a lower risk of incident metabolic syndrome over 25 years among young adults: the CARDIA (Coronary Artery Risk Development in Young Adults) study. Br J Nutr 2014;112:1654-61.

29. Pereira MA, Jacobs DR Jr, Van Horn L, et al. Dairy consumption, obesity, and the insulin resistance syndrome in young adults: the CARDIA Study. JAMA 2002;287:2081-9.

30. LaMonte MJ, Blair SN, Church TS. Physical activity and diabetes prevention. J Appl Physiol 2005;99:1205-13.

31. Sigal RJ, Kenny GP, Wasserman DH, et al. Physical activity/ exercise and type 2 diabetes. Diabetes Care 2004;27:2518-39.

32. Tierney AC, Roche HM. The potential role of olive oil-derived MUFA in insulin sensitivity. Mol Nutr Food Res 2007;51:1235-48.

33. Salas-Salvado J, Martinez-Gonzalez MA, Bullo M, et al. The role of diet in the prevention of type 2 diabetes. Nutr Metab Cardiovasc Dis 2011;21(Suppl 2):B32-48.

34. Perdomo L, Beneit N, Otero YF, et al. Protective role of oleic acid against cardiovascular insulin resistance and in the early and late cellular atherosclerotic process. Cardiovasc Diabetol 2015;14:75.

35. Covas MI, Konstantinidou V, Fito M. Olive oil and cardiovascular health. J Cardiovasc Pharmacol 2009;54:477-82. 